\title{
Los peces de la cueva de Praileaitz I (Deba, Gipuzkoa)
}

\author{
Praileaitz I (Deba, Gipuzkoa) haitzuloaren arrainak \\ The fishes from the cave of Praileaitz I (Deba, Gipuzkoa)
}

PALABRAS CLAVE: Peces, Salmo sp., Prehistoria, País Vasco, Tafonomía, Pesca.
GAKO-HITZAK: Arrainak, Salmo sp., Euskal Herria, Tafonomia, Arrantza.
KEYWORDS: Fishes, Salmo sp., Prehistory, Basque Country, Taphonomy, Fishing.

Eufrasia ROSELLÓ IZQUIERDO(1), Arturo MORALES MUÑIZ(2)

\section{RESUMEN}

El pequeño tamaño de la colección íctica recuperada en la cueva de Praileaitz I, que abarca desde el Solutrense al Epipaleolítico, seguramente explica el que su diversidad quede circunscrita al más frecuente taxón de las colecciones paleolíticas cantábricas, como es el Género Salmo. Tal limitación taxonómica no parece, en cambio, acorde con la dominancia de las muestras por parte de salmónidos por debajo de los $20 \mathrm{~cm}$ de longitud máxima. Aunque existe una minoría de restos en el umbral de lo que parece factible haya sido pescado por humanos, tal distribución de tallas apunta a un origen mixto de las muestras, donde además de la actividad pesquera parecen haber existido agentes adicionales de acumulación de restos que nos resulta imposible inferir.

\section{LABURPENA}

Praileaitz I-ko haitzuloan berreskuratutako bilduma iktiko txikiak, Solutre alditik Epipaleolito Aro bitartekoak, ziurrenik bere aniztasuna kantabriar Paleolito Aroko bildumetan ohikoena den taxonera mugatuta geratzea azaldu dezake: Salmo generora. Halako muga taxonomikoak ez dirudi ordea, 20 cm-ko luzera maximoaz azpiko izokinen familiako laginen nagusitasunarekin zerikusia duenik. Nahiz eta gizakiek arrantzatuak izan zitekeen aztarna gutxiengo bat egon, halako neurri-banaketak laginen jatorria anitza izan daitekeela pentsarazten du. Alegia, arrantza-jardueraz gain, badirudi, zehaztea ezinezkoak gertatzen zaizkigun aztarna-metaketako agente gehigarriak daudela.

\section{ABSTRACT}

The small size of the fish assemblage retrieved at the cave of Praileaitz I, documenting a sequence that ranges from the Solutrean to the Epipaleolithic, partly explains a taxonomical diversity restricted to the most frequent of the Paleolithic fish taxa from the Cantabrian region, namely the Genus Salmo. Small sample size, on the other hand, runs counterintuitive with the presence of fishes with maximum lengths below $20 \mathrm{~cm}$ that dominate the samples. Although there exists a minority of remains whose size allows one to postulate humans as the responsible accumulating agent, the abundance of these small specimens hints at an heterogeneous origin of the deposits, where other agents, thus far impossible to infer, played a role in the accumulation of the fish remains.

\section{1.- INTRODUCCIÓN}

Tras unos inicios prometedores a finales del siglo pasado, los estudios de peces arqueológicos en el Cantábrico parecen haber languidecido a pesar del interés que supone la pesca durante el Paleolítico Superior europeo (CLEYET-MERLE, 1990). Durante el último lustro los análisis sobre peces prehistóricos cantábricos han comenzado a manifestar un impulso como consecuencia, entre otros, de la introducción de más minuciosos métodos de recuperación. Las lagunas que persisten se nos manifiestan, sobre todo, a nivel cronológico al constatar lo menguado del registro previo al periodo magdaleniense (ROSELLÓ, MORALES, 2014). En este trabajo presentamos una pequeña colección que, aunque con mayoría de hallazgos centrados en las etapas magdaleniense y epipaleolítica, es una de las contadas cantábricas que incorpora asimismo hallazgos ícticos correspondientes al periodo Solutrense.

\section{2.- MATERIAL Y MÉTODOS}

La muestra, compuesta por 31 vértebras, se identificó con la ayuda de la colección comparativa de Arturo Morales Muñiz sita en el Laboratorio de Arqueozoología de la U.A.M. A pesar de que no se realizó una toma de medidas en las piezas, la estimación de la talla se ha llevado a cabo por comparación directa con los ejemplares de dicha colección comparativa. Las tallas se sitúan en todos los casos dentro de un intervalo de $+/-0,5 \mathrm{~cm}$ por lo que deben siempre ser tomadas a título aproximativo. Por lo que se refiere al estudio de huellas, se llevó a cabo tanto una inspección ocular como un análisis con lupa binocular (WILD MZ5) de la superficie de todas las piezas.

(1-2) Laboratorio de Arqueozoología. Dpto. BiologíaUniversidad Autónoma de Madrid. arturo.morales@uam.es 


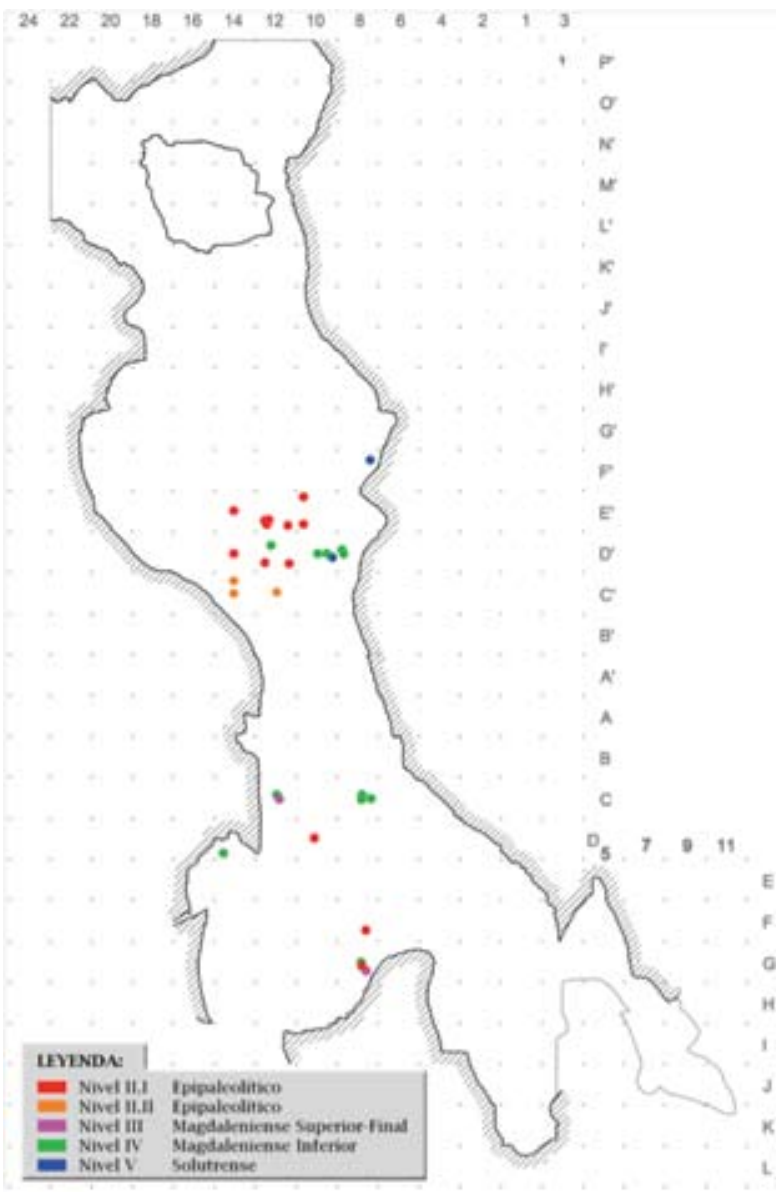

Fig. 1. Planimetría de la excavación con localización de los restos de vértebras por zonas y periodos. / Groundplan of the excavated area with location of the vertebral remains according to zone and period.

\section{3.- RESULTADOS}

Todos los restos estudiados se reparten en tres zonas de la cueva. La mayor concentración se produce en la primera sala interior con un total de 17 piezas, documentándose otras 11 en el vestíbulo y 3 más en el tránsito entre ambos espacios (Fig. 1).

La asignación cronológico-cultural abarca cinco niveles, correspondiendo los niveles II.I y II.II al Epipaleolítico, el nivel III al Magdaleniense Superior-Final, el IV al Magdaleniense Inferior y el nivel $\mathrm{V}$ al Solutrense.

Los dos niveles del paquete Epipaleolítico totalizan 16 restos, 13 de los cuales están asociados con un hogar en cubeta situado en el comienzo de la primera sala interior que incorpora una concentración de 237 conchas marinas. Esta colección está dominada por los bígaros de las especies Littorina obtusata (193 restos) y L. saxatilis (42), apareciendo asimismo un sólo resto de lapa (Patella sp.). Las 3 últimas vértebras aparecieron en el vestíbulo.

Los 11 restos correspondientes al Magdaleniense Inferior se localizan tanto en el vestíbulo (6) como en la primera sala interior de la cueva (5). Los restos del vestí- bulo aparecen relacionados con el hogar situado frente a la entrada de la cueva (Fig. 1).

Del nivel correspondiente al Magdaleniense SuperiorFinal se recuperaron únicamente 2 restos en la zona del vestíbulo, en tanto que las dos vértebras del Solutrense aparecieron dentro de la primera sala interior (Fig. 1).

Todas las vértebras pertenecen a ejemplares de no muy elevado tamaño, siempre por debajo de los $30 \mathrm{~cm}$ de longitud total (LT). Esta talla, que se corresponde con peces en torno a los 250-300 gramos de peso, supone apenas un tercio del total de la muestra (10). Los dos tercios restantes son ejemplares de talla inferior a los $20 \mathrm{~cm}$ LT (apenas $90 \mathrm{~g}$ ) siendo que algunos apenas superarían los $10 \mathrm{~cm}$ LT lo que equivaldría a pesos inferiores a los $50 \mathrm{~g}$.

En ningún caso se han apreciado huellas de termoalteraciones así como tampoco huellas asociadas con actividad humana (cortes, raspados) ni con evidencias de tránsito de las piezas por el tracto digestivo (erosiones).

Todas las piezas representan salmónidos del Género Salmo los cuales son muy difíciles de identificar a especie a través de su anatomía vertebral (Fig. 2). Por el contexto de ubicación del yacimiento en aquellos momentos, con una costa distante unos $4 \mathrm{~km}$, parece más parsimonioso atribuir estos restos a la trucha de río (Salmo trutta fario) que a ejemplares juveniles de reo o trucha marisca (Salmo trutta trutta, forma costera que penetra regularmente en aguas dulces donde pasa las primeras etapas de vida) o salmón (Salmo salar), especie anfídroma y migradora que trasiega regularmente entre el mar y el agua dulce.

Tales especulaciones se ven confirmadas al analizar los anillos de crecimiento (annuli) en los centros vertebrales de algunas de las piezas de mayor tamaño (la colección de piezas en torno a los $30 \mathrm{~cm}$ LT). Como puede apreciarse en la Fig. 3, las piezas que hemos podido analizar presentan un mínimo de 4 anillos que representarían, cuando menos, otros tantos años de vida (el desgaste que acusan los bordes del centro vertebral nos obliga a ser cautos y tomar el quinto año del borde del centro vertebral con mucha cautela). Este hecho se nos antoja capital a efectos de identificación específica. En efecto, si bien en las primeras etapas del desarrollo (e.d., 1-2 años) el crecimiento de los salmónidos europeos no difiere apreciablemente entre especies (p.ej., al alcanzar el segundo año de vida tanto truchas como reos o salmones suelen oscilar entre los 15-22 cm LT para pesos comprendidos entre los 50-100 g), a partir del segundo año, con el descenso al mar, tanto en reos como especialmente en salmones, el crecimiento diverge notablemente del de las truchas de río (FROESE, PAULY, 2014). Así, mientras éstas raramente superan los $50 \mathrm{~cm}$ LT (en torno a 1-1,5 kg), el salmón duplica cómodamente tal talla y la mayoría de los ejemplares tras sólo un año de vida en el mar (esto es, al alcanzar los 3 años) oscilan entre los 50-65 cm LT (1,5-3,5 kg), en tanto que los que alcanzan los 4 años presentan LT comprendidas entre los $70-90 \mathrm{~cm}(4-8 \mathrm{~kg})$, parámetro que a los 5 años se si- 


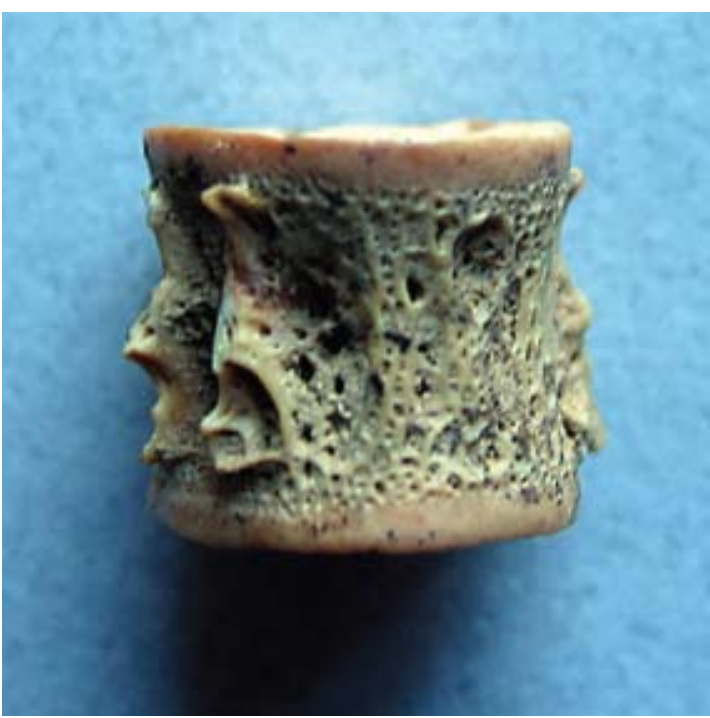

Fig. 2. Vértebra de salmónido en norma dorso-lateral. / Dorso-latera view of a salmonid vertebra.

túa en valores comprendidos entre los 90-105 cm (8-13 $\mathrm{kg}$ ). Así las cosas, parece claro que los salmónidos de mayor talla en Praileaitz I, con 4-5 años de edad (Fig. 3) y tallas en torno a los $30 \mathrm{~cm}$ parecen corresponder con el patrón de crecimiento de la trucha de río. No podemos, sin embargo, aseverar esto con rotundidad por cuanto ocasionalmente (hoy en día no tan raramente) algunos salmones -y también algunos reos- permanecen en agua dulce toda su vida con lo cual la divergencia en su patrón de crecimiento con la trucha de río no sería tan acusada como el que acabamos de referir. Es por ello por lo que preferimos mantener la cautela y dejar los restos de Praileaitz I identificados a nivel de género y no como truchas de río.

\section{4.- DISCUSIÓN Y CONCLUSIONES}

De confirmarse la asignación taxonómica de los restos como pertenecientes a trucha de río, la muestra apuntaría a una asociación piscícola de origen local, en las aguas dulces colindantes con el yacimiento.

La distribución contagiosa de restos que hemos referido (vide supra) parece remitirnos a agentes determinísticos para explicar su presencia en la cueva. Más complicado sería determinar la naturaleza del agente, que parece, en cualquier caso, de carácter biológico (la posibilidad de la existencia de acúmulos naturales, especialmente referidos a mortandades asociadas con crecidas de río quedaría descarta, dada la cota - ca. 50 m s.n.m.- a la que se sitúa la cavidad con respecto al nivel de la ría. En función del contexto habitacional excavado, el agente más probable parece haber sido el ser humano. Tal hipótesis se refuerza con el hecho de que muchas de las vértebras, y en especial todas las de mayor tamaño (recordémoslo, en torno a los $30 \mathrm{~cm}$ de

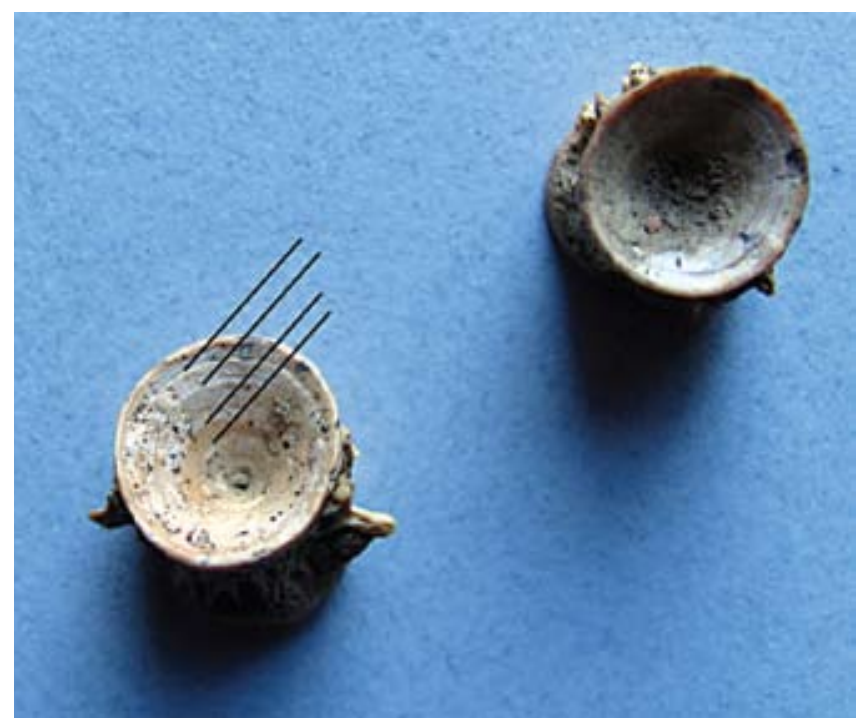

Fig. 3. Vértebras de salmónidos en norma caudal. Las flechas señalan los anillos de crecimiento del centro vertebral. / Caudal views of salmonid vertebrae. Arrows highlight the growth rings of the vertebral centra.

LT) aparecen asociadas con hogares (7 vértebras "grandes" en el hogar en cubeta de la primera sala y otras 3 vértebras en el hogar del vestíbulo). Comoquiera que ambos hogares corresponden a momentos diferentes (Epipaleolítico y Magdaleniense Inferior), tal circunstancia nos apunta a una práctica recurrente de la actividad pesquera en los momentos de la ocupación antrópica. Pero la dominancia de las muestras por parte de ejemplares de muy pequeña talla, cuando no diminutos, no parece encajar con tal práctica pesquera y sí en cambio con la actividad alternativa o complementaria de otros agentes acumuladores, caso de la nutria. La nutria es una especie que puede producir notables acúmulos de huesos de peces como resultado de sus hábitos territoriales y constan situaciones en donde tales depósitos pueden contaminar registros arqueológicos (KRUUK, MOORHOUSE, 1990; NICHOLSON, 2000) Aunque la especie no ha sido detectada en el registro arqueológico de la cueva, ya ha sido invocada como agente acumulador alternativo en el abrigo de Laminak II y la cueva de Aitzbitarte IV (ROSELLÓ, BRINKHUIZEN, 1994; ROSELLÓ, MORALES, 2011).

\section{5.- AGRADECIMIENTOS}

Este trabajo ha sido realizado con ayuda del proyecto HAR 2014-55722-P ("Ictioarqueologia de la Prehistoria cantábrica: Modelos para la caracterización de las primeras pesquerías europeas") del Ministerio de Economía y Competitividad y del proyecto 19438/PI/14 ("Proyecto Gavilanes: Explotación de recursos naturales en el litoral de Mazarrón (Sureste Ibérico) de fines del III milenio a.C. al cambio de Era. Formas, modelos de explotación y derivaciones paleoecológicas") del PROGRAMA SÉNECA 2014. 


\section{6.-BIBLIOGRAFÍA}

CLEYET MERLE, J.J.

1990 La Prehistoire de la Pêche. Editions Errance. Paris.

FROESE, R., PAULY, D. (Ed.).

2014 FishBase. Available at: wuw.fishbase.org (22/10/2014).

KRUUK, H., MOORHOUSE, A.

1990 Seasonal and spatial differences in food selection by otters (Lutra lutra) in Shetland. Journal of Zoology 221(4), 621-637.

NICHOLSON, R.

2000 Otter (Lutra lutra) spraint: An investigation into possible sources of small fishbones at coastal archaeological sites, in: HUNTLEY, J.P. \& STALLIBRASS, S. (eds.). Taphonomy and interpretation. 55-64. (Symposia of the Association of Environmental Archaeology; 14).

ROSELLÓ, E., BRINKHUIZEN, D.

1994 Laminak II / Spain: Alternative taxonomies as approaches to the interpretation of a fish fauna. Offa 51, 401-409.

ROSELLÓ, E., MORALES, A.

2011 Estudio de los peces del yacimiento de Aitzbitarte III (Zona de entrada), en ALTUNA, J., MARIEZKUURRENA, K. \& RíOS, J. Ocupaciones humanas en Aitzbitarte III (País Vasco) 33.600-18.400 BP (Zona de entrada a la cueva). 509-516. (EKOB; 5).

2014 Las ictiofaunas de Santa Catalina (Lekeitio, Bizkaia): un registro singular para la Prehistoria Cantábrica, en BERGANZA GOCHI, E. \& ARRIBAS PASTOR, E. (Coord.). La Cueva de Santa Catalina (Lekeitio): La intervención arqueológica. Restos vegetales, animales y humanos. 161 261. (Kobie BAl; 4). 
ANEXO

Inventario de hallazgos

\begin{tabular}{|c|c|c|c|c|c|c|c|c|c|}
\hline ÁREA & CUADRO & SIGLAS & LECHO & & NIVEL & SECTOR & $\mathbf{x}$ & $\mathbf{Y}$ & $\mathbf{z}$ \\
\hline Vestíbulo & $8 G$ & P.A.8G.209.42 & 6 & $\|$ & Epipaleolítico & & & & 209 \\
\hline Vestíbulo & 10D & P.A.10D.227.29 & 8 & $\|$ & Epipaleolítico & & & & 227 \\
\hline Vestíbulo & $8 \mathrm{~F}$ & P.A.8F.230.84 & 8 & $\|$ & Epipaleolítico & 3 & & & 230 \\
\hline Vestíbulo & $8 G$ & P.A.8G.223.94 & 9 & $\|$ & Epipaleolítico & 3 & & & 223 \\
\hline Vestíbulo & $12 \mathrm{C}$ & P.A.12C.255.24 & 10 & III & Magdaleniense Superior-Final & & & & 255 \\
\hline Vestíbulo & $14 \mathrm{D}$ & P.A.14D.317.22 & 11 & III & Magdaleniense Superior-Final & 1 & 4 & 15 & 317 \\
\hline Vestíbulo & $8 G$ & P.A.8G.259.102 & 11 & IV & Magdaleniense Inferior & $5-8$ & & & 259 \\
\hline Vestíbulo & $12 \mathrm{C}$ & P.A.12C.283.61 & 11 & IV & Magdaleniense Inferior & $5-8$ & & & 283 \\
\hline Vestíbulo & $8 \mathrm{C}$ & P.A.8C.290.21 & 11 & IV & Magdaleniense Inferior & 6 & 95 & 41 & 290 \\
\hline Vestíbulo & $8 \mathrm{C}$ & P.A.8C.287.19 & 11 & IV & Magdaleniense Inferior & 5 & 65 & 50 & 287 \\
\hline Vestíbulo & $8 \mathrm{C}$ & P.A.8C.287.20 & 11 & IV & Magdaleniense Inferior & 5 & 65 & 50 & 287 \\
\hline Pasillo & $14 C^{\prime}$ & P.A.14C'.324.42 & 16 & $11 \mathrm{~b}$ & Epipaleolítico & 8 & & & 324 \\
\hline Pasillo & $14 C^{\prime}$ & P.A.14C'.322.43 & 16 & $11 \mathrm{~b}$ & Epipaleolítico & 5 & & & 322 \\
\hline Pasillo & $12 C^{\prime}$ & P.A.12C'.306.44 & 16 & $11 \mathrm{~b}$ & Epipaleolítico & & & & 306 \\
\hline $1^{\text {a }}$ sala interior & $12 \mathrm{E}^{\prime}$ & P.A.12E'.313.26 & 3 & $\|$ & Epipaleolítico & 1 & & & 313 \\
\hline $1^{\text {a }}$ sala interior & $12 \mathrm{E}^{\prime}$ & P.A.12E'.313.25 & 3 & $\|$ & Epipaleolítico & 1 & & & 313 \\
\hline $1^{\text {a }}$ sala interior & $12 \mathrm{E}^{\prime}$ & P.A.12E'.315.24 & 3 & $\|$ & Epipaleolítico & & & & 315 \\
\hline $1^{\text {a }}$ sala interior & $12 \mathrm{E}^{\prime}$ & P.A.12E'.323.27 & 4 & $\|$ & Epipaleolítico & 1 & & & 323 \\
\hline $1^{\text {a }}$ sala interior & $10 \mathrm{E}^{\prime}$ & P.A.10E'.311.34 & 4 & $\|$ & Epipaleolítico & 1 & & & 311 \\
\hline $1^{\text {a }}$ sala interior & 10E' & P.A.10E'.321.33 & 4 & $\|$ & Epipaleolítico & 7 & & & 321 \\
\hline $1^{\text {a }}$ sala interior & 14E' & P.A.14E'.342.12 & 5,6 & $\|$ & Epipaleolítico & & & & 342 \\
\hline $1^{\text {a }}$ sala interior & 12D' & P.A.12D'.325.74 & 6 & $\|$ & Epipaleolítico & 1 & & & 325 \\
\hline $1^{\text {a }}$ sala interior & $12 D^{\prime}$ & P.A.12D'.315.75 & 6 & $\|$ & Epipaleolítico & 3 & & & 315 \\
\hline $1^{\text {a }}$ sala interior & $14 D^{\prime}$ & P.A.14D'.332.25 & 6 & $\|$ & Epipaleolítico & 5 & & & 332 \\
\hline $1^{\text {a }}$ sala interior & 10D' & P.A.10D'.325.31 & 7 & IV & Magdaleniense Inferior & & & & 325 \\
\hline $1^{\text {a }}$ sala interior & 10D' & P.A.10D'.323.30 & 7 & IV & Magdaleniense Inferior & 6 & & & 323 \\
\hline $1^{\text {a }}$ sala interior & 8D' & P.A.8D'.322.38 & 7 & IV & Magdaleniense Inferior & 4 & & & 322 \\
\hline $1^{\text {a }}$ sala interior & 8D' & P.A.8D'.322.37 & 7 & IV & Magdaleniense Inferior & 4 & & & 322 \\
\hline $1^{\text {a }}$ sala interior & $12 D^{\prime}$ & P.A.12D'.341.28 & 8 & IV & Magdaleniense Inferior & & 96 & 50 & 341 \\
\hline $1^{\text {a }}$ sala interior & 10D' & P.A.10D'.334.61 & 13 & V & Solutrense & 3 & & & 334 \\
\hline $1^{\text {a }}$ sala interior & $8 F^{\prime}$ & P.A.8F'.35.31 & 14 & V & Solutrense & 9 & 67 & 99 & 338 \\
\hline
\end{tabular}


